\title{
Luminosity Bias. II. The Cosmic Web of the First Stars
}

\author{
R. Barkana ${ }^{1,2}$ \\ ${ }^{1}$ Raymond and Beverly Sackler School of Physics and Astronomy, Tel Aviv University, Tel Aviv 69978, Israel \\ ${ }^{2}$ Email: barkana@wise.tau.ac.il
}

(Received December 25, 2012; Accepted March 26, 2013; Online Publication May 12, 2013)

\begin{abstract}
Understanding the formation and evolution of the first stars and galaxies represents one of the most exciting frontiers in astronomy. Since the universe was filled with neutral hydrogen at early times, the most promising method for observing the epoch of the first stars is using the prominent $21-\mathrm{cm}$ spectral line of the hydrogen atom. Current observational efforts are focused on the reionisation era (cosmic age $t \sim 500 \mathrm{Myr}$ ), with earlier times considered much more challenging. However, the next frontier of even earlier galaxy formation $(t \sim 200 \mathrm{Myr})$ is emerging as a promising observational target. This is made possible by a recently noticed effect of a significant relative velocity between the baryons and dark matter at early times. The velocity difference suppresses star formation, causing a unique form of early luminosity bias. The spatial variation of this suppression enhances large-scale clustering and produces a prominent cosmic web on 100 comoving Mpc scales in the 21-cm intensity distribution. This structure makes it much more feasible for radio astronomers to detect these early stars, and should drive a new focus on this era, which is rich with little-explored astrophysics.
\end{abstract}

Keywords: cosmology: theory - galaxies: formation - galaxies: high-redshift - intergalactic medium - large-scale structure of Universe

\section{INTRODUCTION}

\subsection{Cosmic reionisation and the first stars}

Galaxies around us have been mapped systematically out to a redshift of $z \sim 0.3$ by recent large surveys (Aihara et al. 2011; Colless et al. 2001). The observed galaxy distribution shows a large-scale filament-dominated 'cosmic web' pattern that is reproduced by cosmological numerical simulations (Springel, Frenk, \& White 2006). This structure is well understood theoretically (Bond, Kofman, \& Pogosyan 1996) as arising from the distribution of the primordial density fluctuations, which drove hierarchical structure formation in the early universe. Recent observations have been pushing a new frontier of early cosmic epochs, with individual bright galaxies detected reliably from as early as $z=7.2$ (Ono et al. 2012), which corresponds to $t \sim 750 \mathrm{Myr}$ after the Big Bang.

The refurbished Hubble Space Telescope (HST) and the Spitzer Space Telescope have together provided increasingly detailed spectral information on populations of $z \sim 6$ galaxies, making possible robust determinations of their stellar masses, though substantial uncertainties remain in the derived stellar ages and, thus, their star formation histories (Ono et al. 2010; Schaerer \& de Barros 2010; Curtis-Lake et al. 2013). The James Webb Space Telescope (JWST), planned for launch in 2018, will have a 6.5-m mirror and focus on in- frared wavelengths (Gardner et al. 2006). This should allow it to reach much higher redshifts, detecting dwarf galaxies and perhaps supernovae or gamma-ray burst afterglows.

A major goal of current and upcoming observations is to probe the era of cosmic reionisation. Ever since the discovery that the intergalactic gas throughout the Universe is highly ionised (Gunn \& Peterson 1965), astronomers have been searching for the period when the hydrogen was ionised for a second time after it became neutral at cosmic recombination. This reionisation is the most recent cosmic phase transition, whereby the gas was heated and ionised throughout the universe, affecting subsequent galaxy formation and potentially detectable through its effect on a large range of observations. While the highest-redshift galaxies may be probing the late stages of reionisation, there is also a global constraint from the re-scattering of some of the photons of the cosmic microwave background (CMB) by the freshly created plasma. According to the WMAP and Planck CMB satellites, the bulk of cosmic reionisation occurred at $z \sim 11$ (Hinshaw et al. 2012; Ade et al. 2013).

While this observational progress has been remarkable, theory suggests that a rich, varied history of early galaxy formation still remains to be discovered. In particular, current and planned telescopes can directly detect early galaxies only if they are particularly massive or contain a bright, rare event. However, it is theoretically expected that the bulk of 
the early stars formed in a large number of very small galactic units, which would be difficult to observe individually. Even the requirement of cosmic reionisation likely implies the existence of smaller galaxies than currently observed (e.g., Robertson et al. 2013). Going to even earlier times, high-resolution numerical simulations suggest that the truly earliest stars formed within $\sim 10^{6} \mathrm{M}_{\odot}$ dark matter haloes (Abel, Bryan, \& Norman 2002; Bromm, Coppie, \& Larson 1999). Because this characteristic mass of early galactic host haloes is so small, constraining their abundance observationally would probe primordial density fluctuations on $\sim 20 \mathrm{kpc}$ scales, an order of magnitude below current constraints. This could lead to new limits on models with suppressed smallscale power such as warm dark matter (Barkana, Haiman, \& Ostriker 2001).

The first-star simulations mentioned above can only follow small cosmic volumes, and thus begin to form stars much later than in the real universe (since the simulations do not sample the overdense regions that are the sites of the earliest star formation), but analytical methods show that the very first stars must have formed at $z \sim 65$ (age $t \sim 35 \mathrm{Myr}$ ) (Naoz, Noter, \& Barkana 2006; Fialkov et al. 2012). The formation of the very first star was a major milestone which ended the dark ages of the universe- the period after cosmic recombination, when the universe was dark except for the fading glow of the CMB. It also marked a transition point from the simplicity of the early universe, which was homogeneous and isotropic except for small fluctuations that can be described by linear perturbation theory. The formation of the first stars initiated a new era of complexity and feedback, whereby the nuclear and radiative processes within stars (on the scale of $10^{11} \mathrm{~cm}$ ) began to affect the global state of the cosmic gas on scales larger by up to 15 orders of magnitude.

\section{$1.221-\mathrm{cm}$ cosmology}

The best hope of observing the bulk population of early stars is via the cosmic radiation fields that they produced. The mean radiation level traces the cosmic star formation rate, while spatial fluctuations reflect the clustering of the underlying sources, and thus the mass of their host haloes. In particular, the hyperfine spin-flip transition of neutral hydrogen $(\mathrm{H} \mathrm{I})$ at a wavelength of $21 \mathrm{~cm}$ is potentially the most promising probe of the gas and stars at early times. Observations of this line at a wavelength of $21 \times(1+z) \mathrm{cm}$ can be used to slice the universe as a function of redshift $z$ and obtain a three-dimensional map of the diffuse $\mathrm{H}$ I distribution within it (Hogan \& Rees 1979), in the previously unexplored era of redshifts $\sim 7-200$.

Absorption or emission by the gas along a given line of sight changes the $21-\mathrm{cm}$ brightness temperature $T_{\mathrm{b}}$, measured relative to the temperature of the background source, which here is the CMB (Madau, Meiksin, \& Rees 1997). The observed $T_{\mathrm{b}}$ is determined by the spin temperature $T_{\mathrm{s}}$, an effective temperature that describes the relative abundance of hydrogen atoms in the excited hyperfine level compared with the ground state. Primordial density inhomogeneities imprinted a three-dimensional power spectrum of $21-\mathrm{cm}$ intensity fluctuations on scales down to $\sim 10 \mathrm{kpc}$ (all sizes comoving), making it the richest data set on the sky (Loeb \& Zaldarriaga 2004). In particular, since $21-\mathrm{cm}$ measurements can in principle reach such small scales and do it over a substantial redshift range, they can access more independent modes, e.g., a factor of $\sim 10^{9}$ more than the $\mathrm{CMB}$, making $21-\mathrm{cm}$ measurements potentially much more sensitive to small levels of non-Gaussianity. The potential yield of $21-\mathrm{cm}$ observations is further enhanced by the expected anisotropy of the 21-cm power spectrum (Barkana \& Loeb 2005a; Nusser 2005; Ali, Bharadwaj, \& Pandey 2005; Barkana 2006a).

The 21-cm signal vanished at redshifts above $z \sim 200$, when the gas kinetic temperature, $T_{\mathrm{k}}$, was close to the $\mathrm{CMB}$ temperature, $T_{\gamma}$. Subsequently, the gas cooled adiabatically, faster than the $\mathrm{CMB}$, and atomic collisions kept the spin temperature $T_{\mathrm{s}}$ of the hyperfine level population below $T_{\gamma}$, so that the gas appeared in 21-cm absorption (Scott \& Rees 1990). As the Hubble expansion continued to rarefy the gas, radiative coupling of $T_{\mathrm{s}}$ to $T_{\gamma}$ started to dominate over the collisional coupling of $T_{\mathrm{s}}$ to $T_{\mathrm{k}}$ and the $21-\mathrm{cm}$ signal began to diminish.

Once stars began to form, their radiation produced feedback on the intergalactic medium (IGM) and on other newly forming stars, and substantially affected the $21-\mathrm{cm}$ radiation. The first feedback came from the ultraviolet (UV) photons produced by stars between the Ly $\alpha$ and Lyman limit wavelengths. These photons propagated freely through the universe, redshifted or scattered into the $\operatorname{Ly} \alpha$ resonance, and coupled $T_{\mathrm{s}}$ to $T_{\mathrm{k}}$ once again (Madau et al. 1997) through the Wouthuysen-Field (Wouthuysen 1952; Field 1958) effect by which the two hyperfine states are mixed through the absorption and re-emission of a Ly $\alpha$ photon. Meanwhile, Lyman-Werner (LW) photons dissociated molecular hydrogen and eventually ended the era of primordial star formation driven by molecular cooling (Haiman, Rees, \& Loeb 1997), leading to the dominance of larger haloes. X-ray photons also propagated far from the emitting sources and began early on to heat the gas (Madau et al. 1997). Once $T_{\mathrm{s}}$ grew larger than $T_{\gamma}$, the gas appeared in 21-cm emission over the CMB level. Emission of UV photons above the Lyman limit by the same galaxies initiated the process of cosmic reionisation by creating ionised bubbles in the neutral gas around these galaxies. See Figure 1 for a brief summary of early cosmic history.

Several arrays of low-frequency radio telescopes are currently being constructed in order to detect the $21-\mathrm{cm}$ fluctuations from cosmic reionisation. Current efforts include the Murchison Wide-field Array (MWA; Bowman, Morales, \& Hewitt 2009), the Low Frequency Array (LOFAR; Harker et al. 2010), the Giant Metrewave Radio Telescope (GMRT; Paciga et al. 2011), and the Precision Array to Probe the Epoch of Reionisation (PAPER; Parsons et al. 2010), and early plans have been made for a future Square Kilometre Array (SKA; e.g. Furlanetto, Oh, \& Briggs 2006). Although the expected foregrounds (dominated by Galactic synchrotron) 


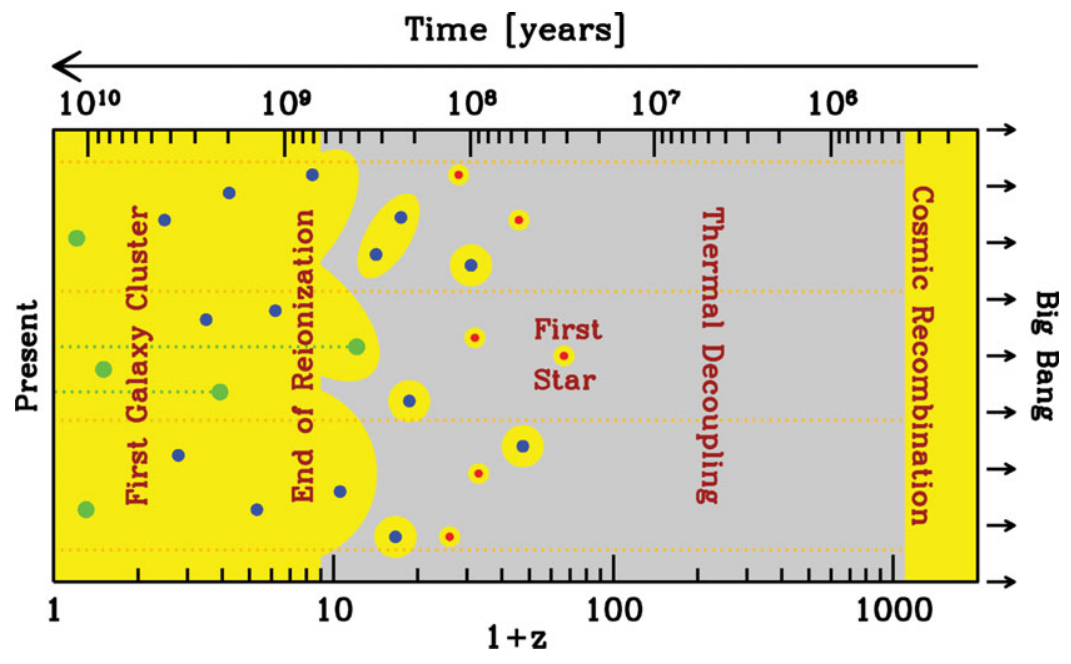

Figure 1. Overview of cosmic history, with the age of the universe shown on the top axis and the corresponding redshift on the bottom axis. Yellow represents ionised hydrogen and grey is neutral. Observers probe the cosmic gas using the absorption of background light (dotted lines) by atomic hydrogen. Stars formed in haloes whose typical size continually grew with time, going from the first generation that formed through molecular-hydrogen cooling (red dots), to the larger galaxies that formed through atomic cooling and likely dominated cosmic reionisation (blue dots), all the way to galaxies as massive as the Milky Way, some of which host bright quasars (green dots). From Barkana (2006b).

are much brighter than the $21-\mathrm{cm}$ signal, they are not expected to include sharp spectral features. Thus, the prospects for extraction of the $21-\mathrm{cm}$ signal (and from it the reionisation history) are quite promising, using the 21-cm power spectrum (Bowman, Morales, \& Hewitt 2007; McQuinn et al. 2006; Barkana 2009) as well as other statistics (Bharadwaj \& Pandey 2005; Furlanetto, Zaldarriaga, \& Hernquist 2004b; Ichikawa et al. 2010; Pan \& Barkana 2012). A different approach is to measure the total sky spectrum and detect the global reionisation signal arising from the overall disappearance of atomic hydrogen (Bowman \& Rodgers 2010; Pritchard \& Loeb 2010; Morandi \& Barkana 2012).

\section{LARGE SPATIAL FLUCTUATIONS IN GALAXY NUMBERS AT HIGH REDSHIFT}

A broad, common thread runs through much of the theoretical development of early galaxy formation over the last decade. The density of galaxies (or stars) varies spatially, with the fluctuations becoming surprisingly large at high redshift, even on quite large cosmological scales (Barkana \& Loeb 2004). This can be understood from the standard theory of galaxy biasing (Press \& Schechter 1974; Kaiser 1984; Bardeen et al. 1986; Cole \& Kaiser 1989; Bond et al. 1991; Mo \& White 1996) as due to the fact that the first galaxies represented rare peaks in the cosmic density field.

As an analogy, imagine searching on Earth for mountain peaks above $5000 \mathrm{~m}$. The 200 such peaks are not at all distributed uniformly but instead are found in a few distinct clusters on top of large mountain ranges. Similarly, in order to find the early galaxies, one must first locate a region with a large-scale density enhancement, and then galaxies will be found there in abundance. The density of stars should thus show strongly biased (i.e. amplified) fluctuations on large scales (Barkana \& Loeb 2004). These large-scale fluctuations, and their effect on everything from feedback to observational predictions, had been previously underestimated, in part because the limited range of scales available to numerical simulations put this topic mostly out of their reach.

This idea first made a major impact on studies of cosmic reionisation. In Barkana \& Loeb (2004) we argued that the typical sizes of $\mathrm{H}$ II bubbles during reionisation should be around 10 or $20 \mathrm{Mpc}$ (see Figure 2), while many numerical simulations of reionisation at the time followed a total box below this size. Further analytical models (Furlanetto, Zaldarriaga, \& Hernquist 2004a) and large-scale numerical simulations (Ciardi, Ferrara, \& White 2003; Zahn et al. 2007; Mellema et al. 2006; Santos et al. 2008) have indeed demonstrated the dominance of large bubbles due to large groups of strongly clustered galaxies. This has helped motivate the large number of observational efforts currently underway, since large-scale fluctuations are easier to detect (as they do not require high angular resolution).

The same idea of large-scale fluctuations in galaxy numbers soon found an important application in a different regime. In Barkana \& Loeb (2005b), we showed that fluctuations in the galaxy number density cause fluctuations even in the intensity of long-range radiation, leading to a new source of 21-cm fluctuations. Specifically, we considered the background of $\operatorname{Ly} \alpha$ radiation. As mentioned above, the spin temperature of hydrogen atoms in the IGM can be coupled to the gas temperature indirectly through the Wouthuysen-Field effect, which involves the absorption of Ly $\alpha$ photons. While it was previously known (Madau et al. 


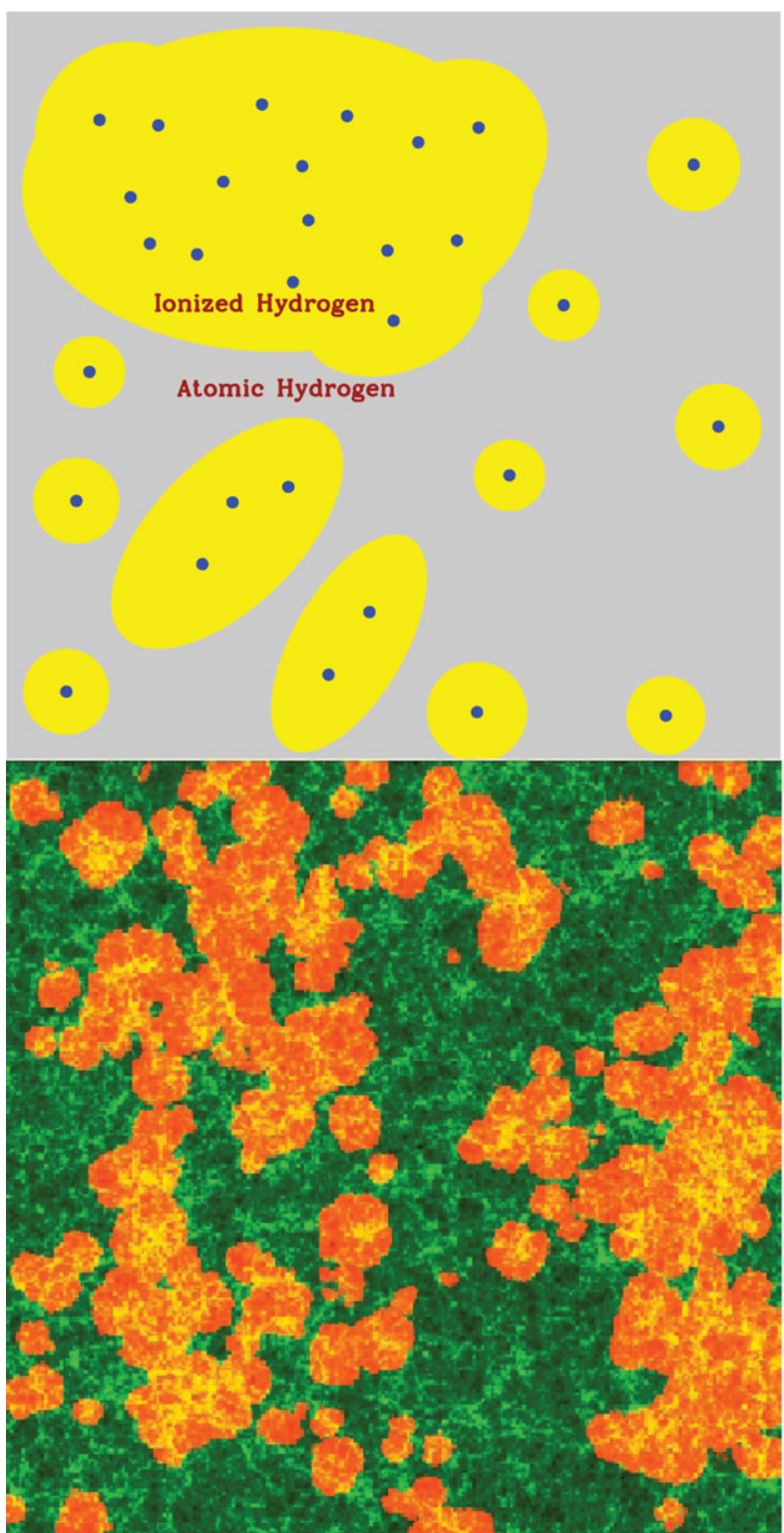

Figure 2. During reionisation, the ionised bubbles created by clustered groups of galaxies (Barkana \& Loeb 2004) imprinted a signature in the power spectrum of 21-cm fluctuations (Furlanetto et al. 2004a). The illustration (top panel, from Barkana 2006b) shows how regions with large-scale overdensities form large concentrations of galaxies (dots) whose ionising photons produce large ionised bubbles. At the same time, other large regions have a low density of galaxies and are still mostly neutral. A similar pattern has been confirmed in large-scale numerical simulations of reionisation (e.g. bottom panel, showing a two-dimensional slice from a $150-\mathrm{Mpc}$ simulation box; Mellema et al. 2006).

1997; Chen \& Miralda-Escudé 2004) that this Ly $\alpha$ coupling likely occurred in the IGM due to Ly $\alpha$ photons emitted by early stars at $z \sim 20-30$, this radiation background had been assumed to be uniform. This intuition was based on the fact that each atom sees $\operatorname{Ly} \alpha$ radiation from sources as far away as $300 \mathrm{Mpc}$. However, we showed that relatively large, potentially observable, $21-\mathrm{cm}$ fluctuations are generated during the era of initial Ly $\alpha$ coupling, for two reasons: galaxy fluc- tuations are significant even on scales of the order of 100 $\mathrm{Mpc}$, and also a significant fraction of the Ly $\alpha$ flux received by each atom comes from relatively nearby sources. Since a relatively small number of galaxies contributed to the flux seen at any given point, we found that Poisson fluctuations could be significant as well, producing correlated $21-\mathrm{cm}$ fluctuations. If observed, we argued, this new predicted signal would not only constitute the first detection of these early galaxies, but the shape and amplitude of the resulting $21-\mathrm{cm}$ power spectrum would also probe their average properties.

This has led to a variety of follow-up work, including more precise analyses of the atomic cascades of Lyman-series photons (Hirata 2006; Pritchard \& Furlanetto 2006) and a calculation of heating due to an inhomogeneous X-ray background (Pritchard \& Furlanetto 2007) that followed a similar approach and found somewhat larger resulting $21-\mathrm{cm}$ fluctuations. We have also predicted a significant boost in the 21$\mathrm{cm}$ power spectrum from Ly $\alpha$ fluctuations (Naoz \& Barkana 2008) due to the repeated scattering of the photons from stars on their way to the hydrogen atoms (see also Chuzhoy, Alvarez, \& Shapiro 2006; Semelin, Combes, \& Baek 2007).

\section{THE NEW GAME IN TOWN: RELATIVE MOTION BETWEEN THE BARYONS AND DARK MATTER}

As noted above, current observational efforts in $21-\mathrm{cm}$ cosmology (and high-redshift astronomy more generally) are focused on the reionisation era (redshift $z \sim 10$ ), with earlier times considered much more difficult to observe. This has begun to change as a result of recent new predictions that suggest that the pre-reionisation, $z \sim 20$ era of even earlier galaxies is primed for observational exploration. This is made possible by a recently noticed effect on early galaxy formation that had been previously neglected.

Up until recently, studies of early structure formation were based on initial conditions from linear perturbation theory. However, there is an important effect missing from this treatment (Tseliakhovich \& Hirata 2010). At early times, the electrons in the ionised gas scattered strongly with the thenenergetic CMB photons, so that the baryons moved together with the photons in a strongly coupled fluid. On the other hand, the motion of the dark matter was determined by gravity, as it did not otherwise interact with the photons. Thus, the initial inhomogeneities in the universe led to the gas and dark matter having different velocities. When the gas recombined at $z \sim 1100$, it was moving relative to the dark matter, with a relative velocity that varied spatially. The root-mean-square value at recombination was $\sim 30 \mathrm{~km} \mathrm{~s}^{-1}$, which is supersonic (Mach number 5).

Figure 3 shows the contribution of fluctuations on various scales to the variance of the velocity difference. This highlights two important properties of this relative motion. First, there is no contribution from small scales, so that the relative velocity is uniform in patches up to a few Mpc in size; the velocity is generated by larger-scale modes, up to $\sim 200 \mathrm{Mpc}$ 


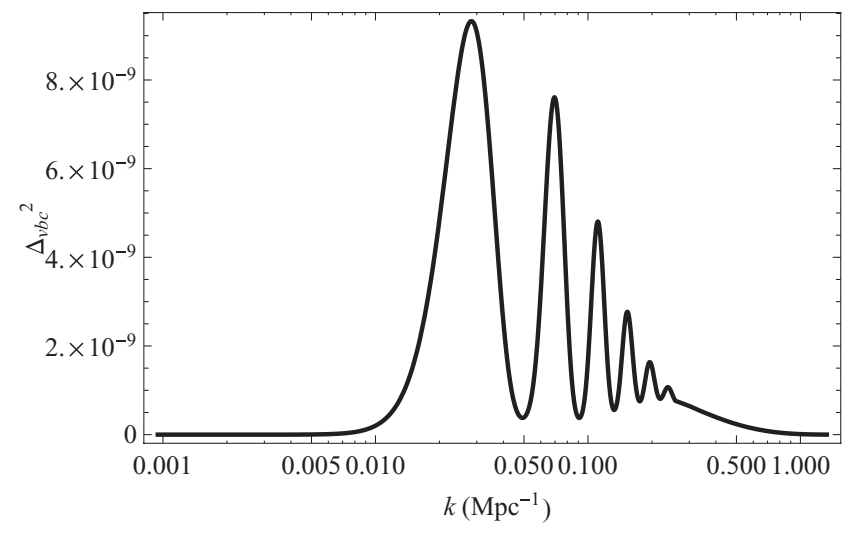

Figure 3. The contribution of various scales to the mean squared velocity difference between the baryons and dark matter (at the same position) at recombination. The contribution per $\log k$ of fluctuations at wavenumber $k$ is shown vs $k$. From Tseliakhovich \& Hirata (2010).

in wavelength. The uniformity on small scales is critical as it allows a separation of scales between the spatial variation of the velocity (on large scales) and galaxy formation (on small scales). Each individual high-redshift mini-galaxy forms out of a small region ( $\sim 20 \mathrm{kpc}$ for a $10^{6} \mathrm{M}_{\odot}$ halo) that can be accurately approximated as having a uniform, local baryonic wind; the relative velocity is thus also referred to as the 'streaming velocity'. The second important feature of Figure 3 is the strong baryon acoustic oscillation (BAO) signature. Arising from the acoustic oscillations of the photon-baryon fluid before recombination, this strong BAO signature is a potential observational fingerprint of the effect of this relative motion, as is further detailed below.

This relative motion effect is not in itself a surprise, but before 2010 it had not been noticed that this effect was dropped within the standard approach. The standard initial conditions for both analytical calculations and numerical simulations have been generated based on linear perturbation theory, in which each $k$ mode evolves independently. Indeed, the relative velocity is negligible if any single scale is considered. However, it is important as an effect of large scales (which contribute to the velocity difference) on small scales (which dominate early galaxy formation). Specifically, the relative motion makes it harder for small-scale overdensities in the dark matter to gravitationally accrete the streaming gas. Now, observing such small-scale fluctuations directly would require far higher resolution than is currently possible. Nonetheless, this effect is immensely important because of the effect on star formation. Since stellar radiation strongly affects $21-\mathrm{cm}$ emission from the surrounding IGM, 21-cm cosmology offers an indirect probe of the relative velocity effect.

The first effect of the streaming velocity on haloes to be analysed was the suppression of the abundance of haloes (Tseliakhovich \& Hirata 2010). Since the baryons do not follow the dark matter perturbations as closely as they would without the velocity effect, linear fluctuations are suppressed on small scales (where the gravitationally induced veloci- ties are comparable to or smaller than the relative velocity). According to the standard theoretical models for understanding the abundance of haloes as a function of mass (Press \& Schechter 1974; Bond et al. 1991), this should result in a reduction of the number density of high-redshift haloes of mass up to $\sim 10^{6} \mathrm{M}_{\odot}$ (Tseliakhovich \& Hirata 2010), a mass range that is expected to include most of the star-forming haloes at early times.

Dalal, Pen, \& Seljak (2010) next pointed out a second effect, namely that separately from the effect on the number of haloes that form, the relative velocity also suppresses the gas content of each halo that does form. They also claimed that this second effect results in $2 \mathrm{mK}$, large-scale 21-cm fluctuations during Ly $\alpha$ coupling, with a power spectrum showing a strong BAO signature due to the streaming velocity effect. These conclusions were later shown to be qualitatively on the mark but invalid quantitatively. In particular, we showed (Tseliakhovich, Barkana, \& Hirata 2011; Fialkov et al. 2012) that the gas-content effect is a minor one on star-forming haloes, and is mainly important for the lower-mass gas minihaloes that do not form stars. Also, a 2-mK signal would in any case be too weak to be of major interest to the observers (see later).

Meanwhile, many groups began to run small-scale numerical simulations that follow individual collapsing haloes subject to the streaming velocity (Maio, Koopmans, \& Ciardi 2011; Stacy, Bromm, \& Loeb 2011; Greif et al. 2011; O'Leary \& McQuinn 2012; McQuinn \& O'Leary 2012; Naoz, Yoshida, \& Gnedin 2012; Naoz, Yoshida, \& Gnedin 2013). In particular, the simulations of Stacy et al. (2011) and Greif et al. (2011) indicated the presence of a third effect, i.e. that the relative velocity substantially increases the minimum halo mass for which stars can form from gas that cools via molecular hydrogen cooling. We found (Fialkov et al. 2012) a fit to these simulation results, and thus developed a general model that includes the effect of density as well as all three effects of the velocity on star formation.

Figure 4 illustrates some of the results of the numerical simulation studies of the effect of the streaming velocity on galaxy formation. As expected, a larger velocity suppresses gas accretion more strongly, in particular reducing the amount of dense gas at the centers of haloes. But beyond just this general trend, the relative velocity effect gives rise to very interesting dynamics on small scales. It disrupts gas accretion in an asymmetric way, so that filaments of accreting gas are disrupted more easily if they are perpendicular to the local wind direction. In addition, haloes that form in regions of relatively high velocity develop supersonic wakes as they move through the wind.

\section{DETECTING THE FIRST STARS AT REDSHIFT 20}

While numerical simulations are the best, most accurate method for studying the small-scale effects of the relative velocity, they are unable to simultaneously cover large volumes. 


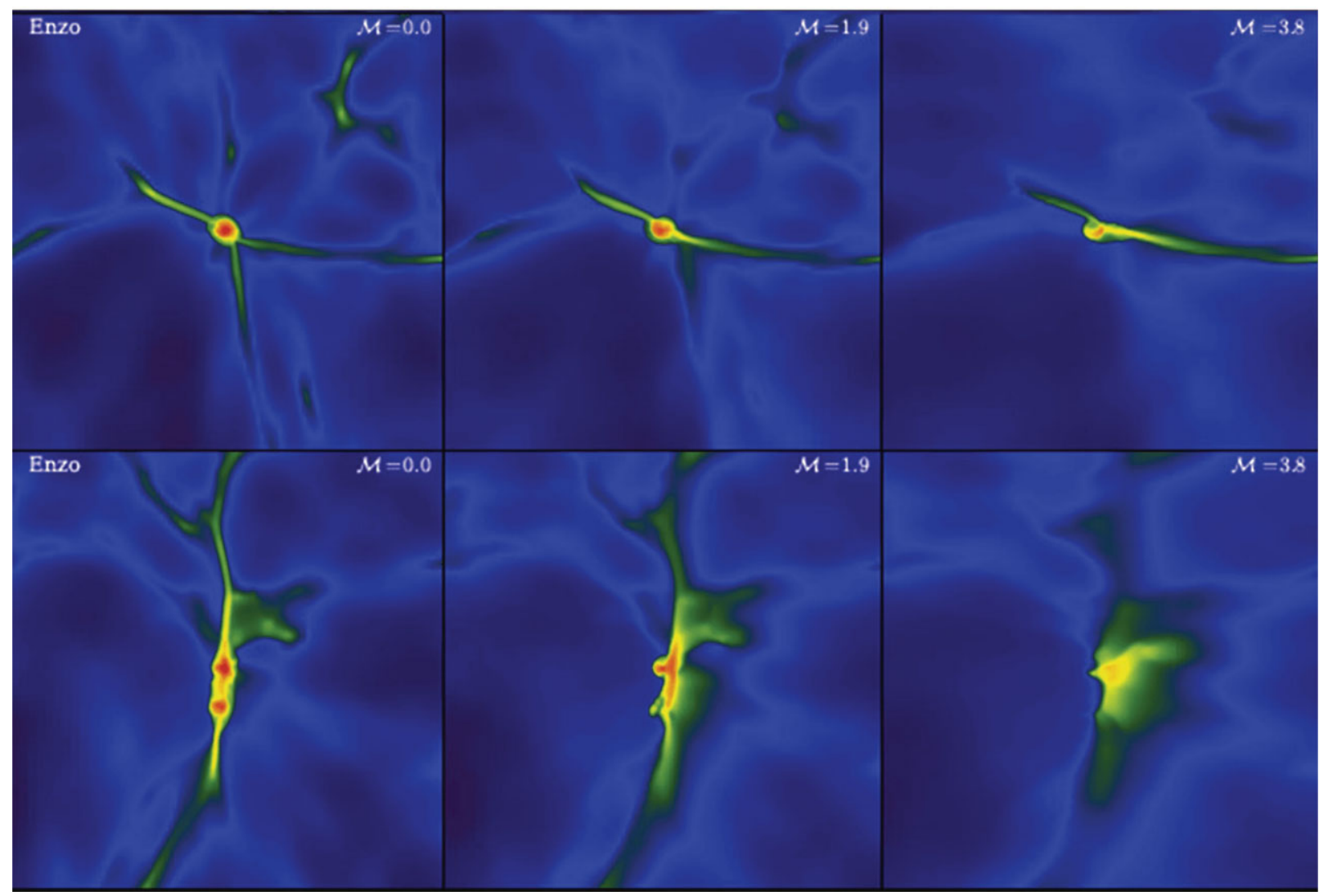

Figure 4. Effect of relative velocity on individual haloes, from numerical simulations (including gravity and hydrodynamics). The colours indicate the gas density, which ranges from $10^{-26}$ (blue) to $10^{-23} \mathrm{~g} \mathrm{~cm}^{-3}$ (red). Two haloes are shown at $z=20$, with a total halo mass of $2 \times 10^{6} \mathrm{M}_{\odot}$ (top) or $8 \times 10^{5} \mathrm{M}_{\odot}$ (bottom). Panels show the result for gas initially moving to the right with a relative velocity of 0 (left), 1 (middle), or 2 (right) in units of the root-mean-square value of the relative velocity at $z=20$. $\mathcal{M}$ indicates the corresponding Mach number at $z=20$. From O'Leary \& McQuinn (2012).

Simulations that successfully resolve the tiny mini-galaxies that dominated star formation at early times are limited to $\sim 1 \mathrm{Mpc}$ volumes, and cannot explore the large cosmological scales that might be accessible to $21-\mathrm{cm}$ observations (which are currently limited to low resolution).

On the other hand, analytical calculations are limited to linear (plus sometimes weakly non-linear) scales, and thus cannot directly probe the non-linear astrophysics of halo and star formation. Even if the results of simulations are incorporated within them, analytical approaches assume small fluctuations and linear bias (i.e., that the distribution of stars is a proportionally amplified version of that of the underlying density), assumptions that break down in the current context, where the stellar density varies by orders of magnitude on scales of a few Mpc. Even on $100 \mathrm{Mpc}$ scales, fluctuations in the gas temperature are as large as order unity (see later). There are also additional non-linear relationships in this problem such as the dependence of 21- $\mathrm{cm}$ temperature on gas temperature. Thus, linear, analytical calculations can only yield rough estimates, even for the large-scale fluctuations.

Thus, the best way to generate observable predictions from this era of early galaxies is with a hybrid method that com- bines linear theory on large scales with the results of numerical simulations on small scales. We recently developed such a hybrid method and used it to produce the first realistic, three-dimensional images of the expected large-scale distribution of the first stars and the resulting 21-cm emission (Visbal et al. 2012). In our approach, we built upon previous hybrid methods used for high-redshift galaxy formation (Tseliakhovich \& Hirata 2010; Dalal et al. 2010; Mesinger, Furlanetto, \& Cen 2011). We first used the known statistical properties of the initial density and velocity perturbations to generate a realistic sample universe on large, linear scales. Then, we calculated the stellar content of each pixel on our grid using the overall model that we had developed (Fialkov et al. 2012) to describe the streaming velocity effect on galaxy formation; this includes various analytical models as well as fits to the results of the small-scale numerical simulations.

We assumed standard initial perturbations (e.g. from a period of inflation), where the density and velocity components are Gaussian random fields. Velocities are coherent on larger scales than the density, due to the extra factor of $1 / k$ in the velocity from the continuity equation that relates the two fields. This is clearly apparent in the example shown in Figure 5 of a thin slice of our simulated volume. The density field 

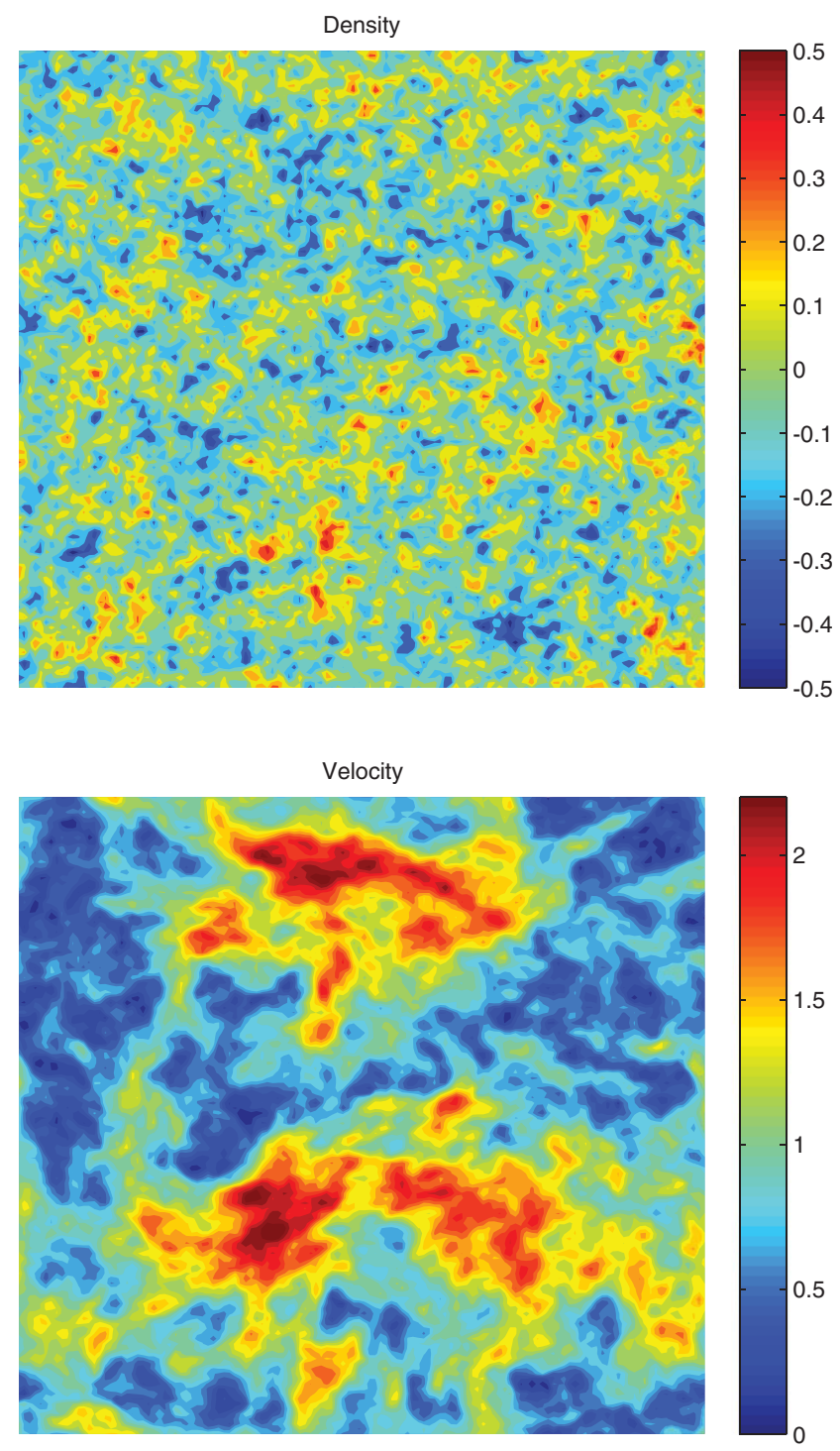

Figure 5. The large-scale density and velocity fields in an example of a slice from a simulated volume $384 \mathrm{Mpc}$ on a side (based on Visbal et al. 2012, but taken from a different box from the one shown in the figures in Visbal et al. 2012, i.e. for a different set of random initial conditions). The thickness of the slice is $3 \mathrm{Mpc}$ (which is also the pixel size of our grid). For the density field (top panel), we show the fractional perturbation relative to the mean, at $z=20$; for the velocity field (bottom), we show the magnitude of the relative motion in units of the root-mean-square value (the map is independent of redshift in these relative units).

fluctuates on relatively small scales, while the velocity field shows a larger-scale cosmic web, with coherent structure on scales of the order of $100 \mathrm{Mpc}$. This means that the largest scales, which are easiest to observe, will be dominated by the pattern due to the velocity effect.

The resulting distribution of stellar density at $z=20$ is shown in Figure 6. Note the large biasing (i.e. amplification of fluctuations) of the stars: density fluctuations ranging up to $\pm 50 \%$ yield (without including the relative velocities) a field of stellar density that varies by over a factor of 20 (both smoothed on the 3-Mpc scale). The velocity effect produces
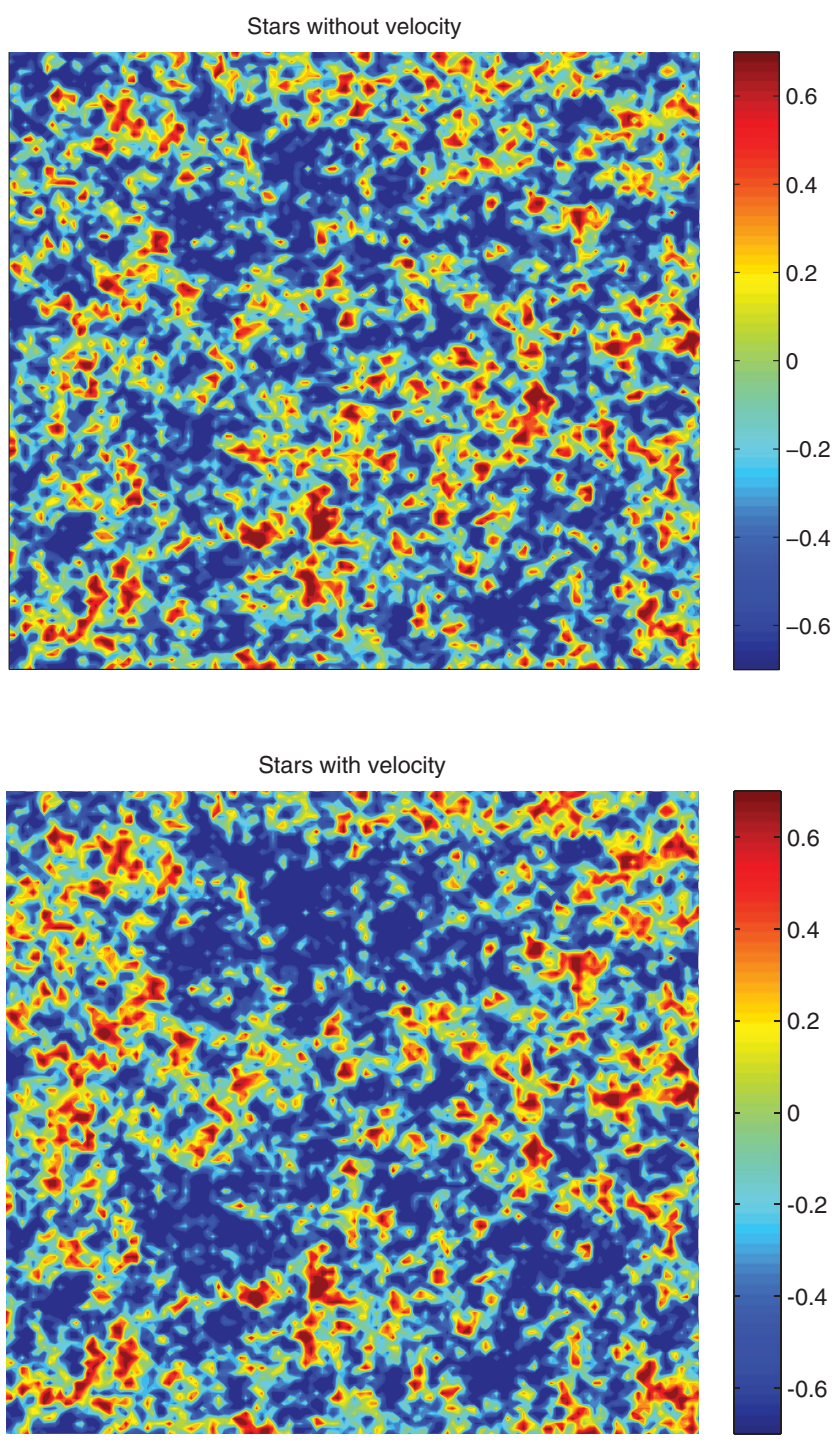

Figure 6. Effect of relative velocity on the number density of stars at redshift 20. For the same slice as in Figure 5, we compare the previous expectation (top panel), including the effect of density only, to the new prediction (bottom), including the effect of the same density field plus that of the relative velocity. The colours correspond to the logarithm of the gas fraction in units of its cosmic mean value in each case.

a more prominent cosmic web on large scales, marked by large coherent regions that have a low density of stars, separated by ribbons or filaments of high star formation. The effect is even more striking at higher redshifts (Figure 7), which substantially alters the feedback environment of the first stars. The various types of radiation that produce feedback spread out to a considerable distance from each source, but this distance is typically not as large as the span of the velocity-induced features. This means that regions of low velocity (and thus high star formation) experience radiative feedback substantially earlier than regions of high velocity (low star formation). Thus, the substantial effect of the velocities on early star formation makes early feedback far more inhomogeneous than previously thought. 


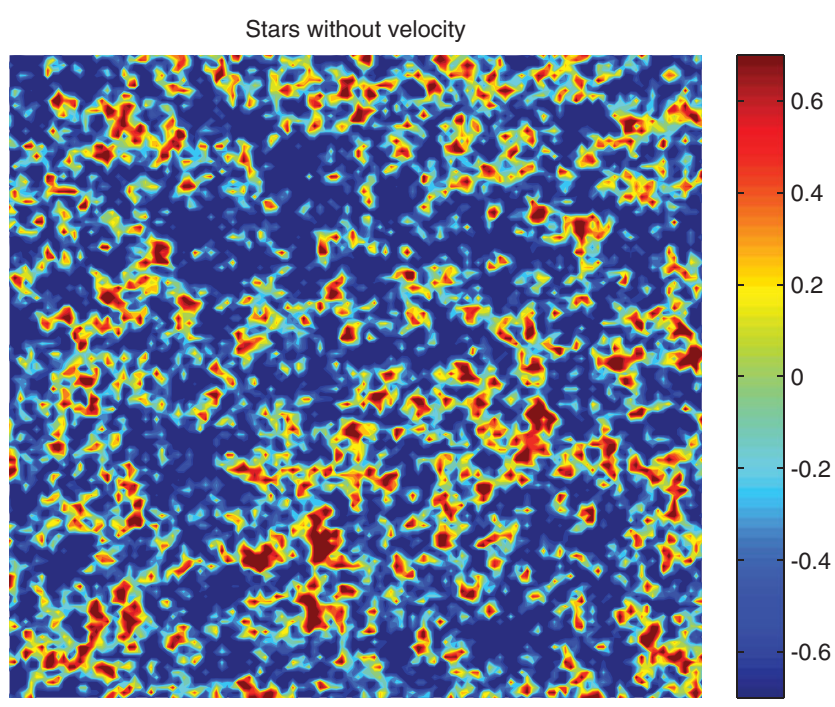

Stars with velocity

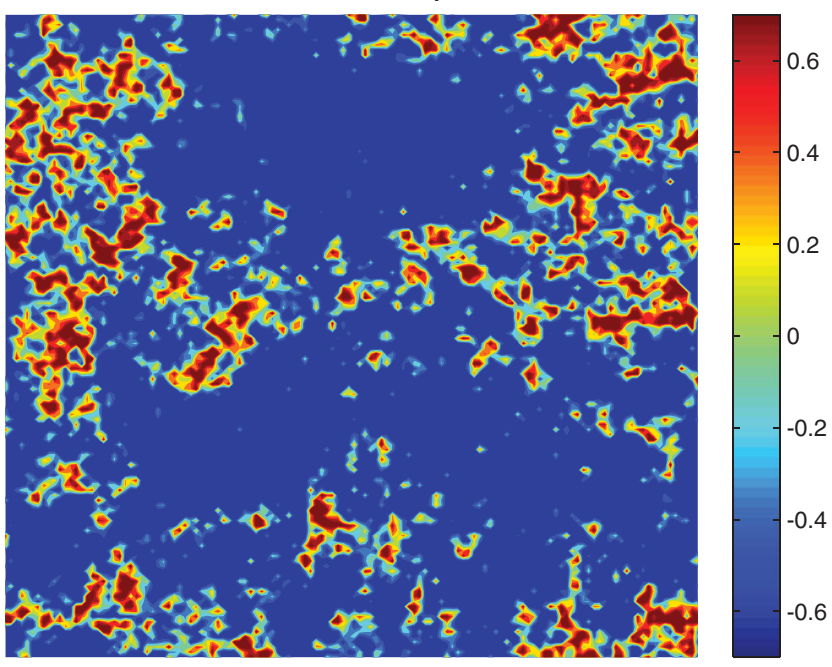

Figure 7. Effect of relative velocity on the number density of stars at redshift 40. For the same slice as in Figure 5, we compare the previous expectation (top panel), including the effect of density only, to the new prediction (bottom), including the effect of the same density field plus that of the relative velocity. The colours correspond to the logarithm of the gas fraction in units of its cosmic mean value in each case. The colour scale spans the same range as in Figure 6 for easy comparison.

Observationally, these degree-scale fluctuations will affect various cosmic radiation backgrounds, and in particular the history of 21-cm emission and absorption, which depends on the timing of the three radiative transitions mentioned in Section 1.2: spin-temperature coupling due to Ly $\alpha$ photons, molecular hydrogen dissociation due to LW photons, and heating due to X-ray photons. Although the timing is still significantly uncertain, the $21-\mathrm{cm}$ coupling due to Ly $\alpha$ radiation is expected to occur rather early, with the X-ray heating fluctuations occurring later and possibly overlapping with significant small-halo suppression due to LW radiation (Visbal et al. 2012). It is most promising to focus on the fluctuations due to X-ray heating around redshift 20 , since
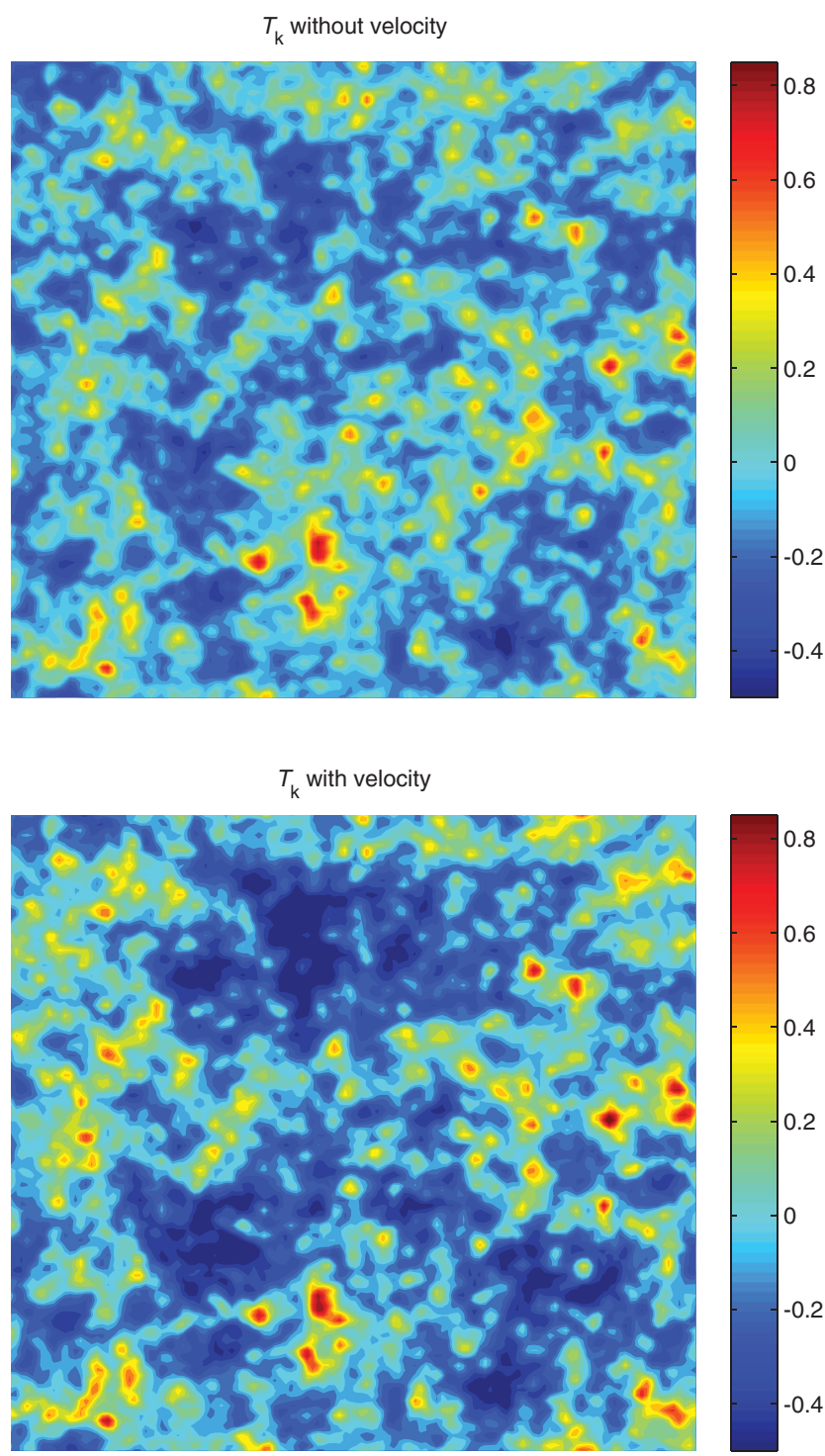

Figure 8. Effect of relative velocity on the gas temperature $T_{\mathrm{k}}$ at redshift 20. For the same slice as in Figure 5, we compare the previous expectation (top panel), including the effect of density only, to the new prediction (bottom), including the effect of density and relative velocity. The colours correspond to the logarithm of the gas (kinetic) temperature in units of the CMB temperature at $z=20$.

these tend to produce larger 21-cm fluctuations (and at lower redshifts that feature lower foreground noise) than the Ly $\alpha$ coupling era (as suggested by previous analyses of these two periods without the relative velocity effect; Barkana \& Loeb 2005b; Pritchard \& Furlanetto 2007). Given the expected timing, we can analyse the heating era assuming that Ly $\alpha$ coupling has already saturated. As for the LW flux, we continue to illustrate here the case of negligible LW feedback (as assumed in Figures 6 and 7), but below we bracket the effect of the LW flux by also considering the opposite limiting case where the LW transition has already saturated.

Figure 8 shows the gas temperature distribution at $z=$ 20 , at the midpoint of the heating transition, i.e., when the 
mean gas temperature is equal to that of the CMB. Regions where the gas moved rapidly with respect to the dark matter (dark red regions, bottom panel of Figure 5) produced fewer stars (dark blue regions, bottom panel of Figure 6) and thus a lower X-ray intensity, leaving large regions with gas that is still colder than the CMB by a factor of several (dark blue regions, bottom panel of Figure 8). The spatial reach of Xrays results in a gas temperature distribution that is smoother than the distribution of stars, and this brings out the effect of large-scale fluctuations and thus highlights the contrast between the effect of density and velocity fluctuations.

During the heating transition, the $21-\mathrm{cm}$ brightness temperature (Figure 9) mainly measures the gas temperature, although it is also proportional to the gas density (and to the square root of $1+z$ ). The form of the dependence, $T_{\mathrm{b}} \propto 1-$ $T_{\mathrm{CMB}} / T_{\text {gas }}$, makes the $21-\mathrm{cm}$ intensity more sensitive to cold gas than to hot gas (relative to the CMB temperature). Thus, the large voids in star formation produced by a high relative velocity lead to prominent $21-\mathrm{cm}$ absorption (dark blue regions, bottom panel of Figure 9) seen on top of the pattern from the effect of density fluctuations. These deep 21-cm cold spots are the main observable signature of the effect of the relative velocity on the first stars. Note that the observed wavelength of this radiation is redshifted by the expansion of the universe to $4.4 \mathrm{~m}$ (corresponding to a frequency of $68 \mathrm{MHz}$ ).

While these figures illustrate the detailed pattern of the effect of relative velocity on the $21-\mathrm{cm}$ intensity distribution, upcoming experiments are expected to yield very noisy maps that likely must be analysed statistically. Figure 10 shows the predicted effect on a key statistic, the power spectrum of the fluctuations in 21-cm intensity (from Visbal et al. 2012). The velocities enhance large-scale fluctuations (blue solid curve compared with red dotted), leading to a flatter power spectrum with prominent baryon acoustic oscillations (reflecting the BAO signature in Figure 3). The signal is potentially observable with a redshift 20 version of current instruments (green dashed curve). If there is strong LW feedback (solid purple curve), then the small galaxies that rely on molecularhydrogen cooling are unable to form; the larger galaxies that dominate in that case are almost unaffected by the streaming velocity, so the $21-\mathrm{cm}$ power spectrum reverts to the densitydominated shape (compare the solid purple and red dotted curves), but it becomes even higher since more massive galactic haloes are even more strongly biased (i.e. clustered).

Thus, regardless of the timing of the LW feedback, the $21-\mathrm{cm}$ power spectrum at the peak of the heating transition should feature large observable fluctuations on large angular scales. Beyond just detection of the signal, only a mild additional accuracy is necessary in order to determine whether feedback has suppressed star formation in the smallest haloes. If it has not, then the velocity effect produces strong BAOs on top of a flattened power spectrum, in particular raising it by a factor of 4 on large scales $(k=$ $0.05 \mathrm{Mpc}^{-1}$, wavelength $130 \mathrm{Mpc}$, observed angle 2/3 of a degree) where experimental sensitivity is optimal. If this
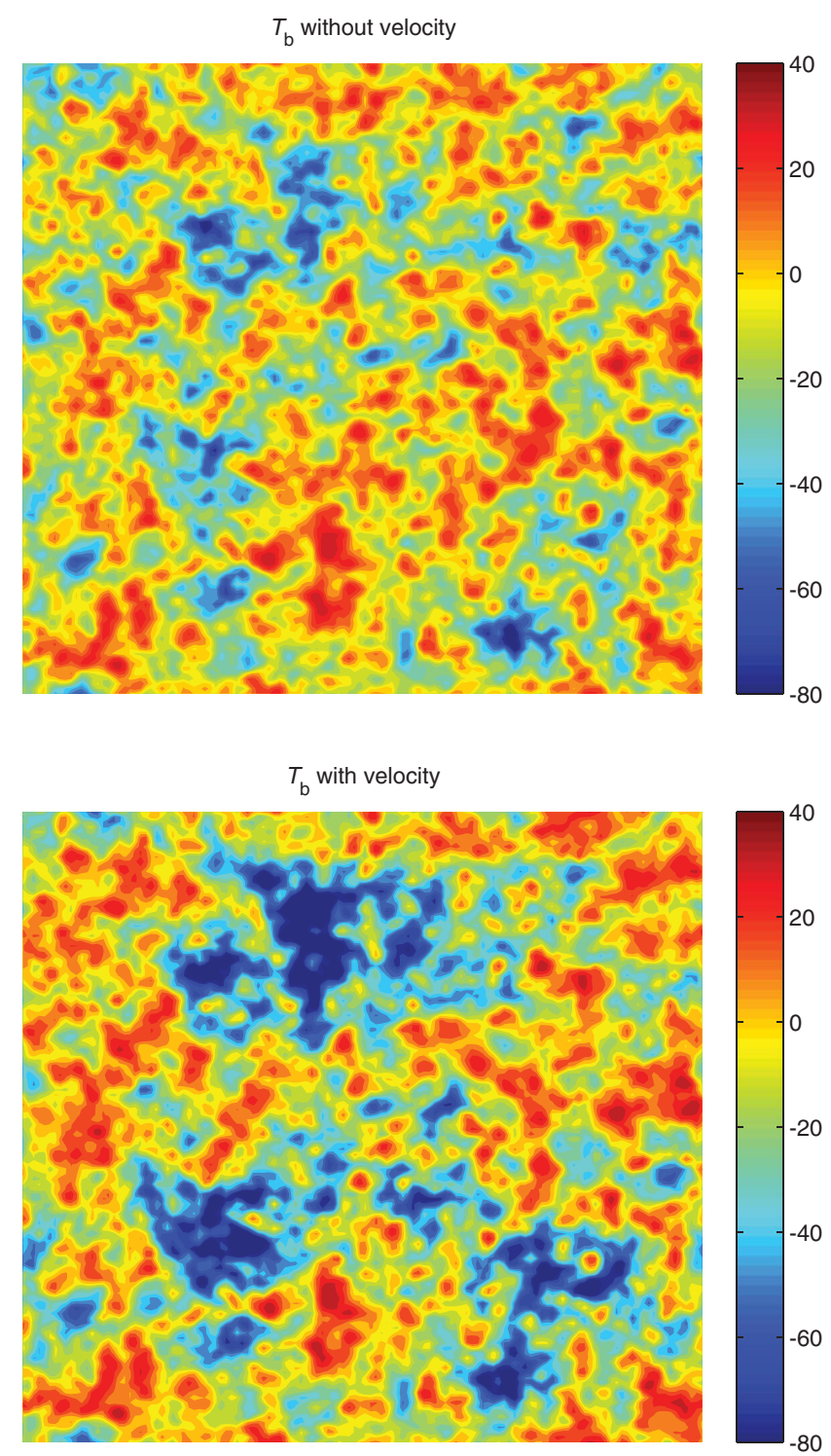

Figure 9. Effect of relative velocity on the redshift $2021-\mathrm{cm}$ brightness temperature $T_{\mathrm{b}}$ (which measures the observed intensity of radio waves emitted by intergalactic hydrogen atoms at $21 \mathrm{~cm}$ ). For the same slice as in Figure 5 , we compare the previous expectation (top panel), including the effect of density only, to the new prediction (bottom), including the effect of density and relative velocity. The colours correspond to the $21-\mathrm{cm}$ brightness temperature in millikelvin units.

characteristic shape is observed, it would confirm that million mass haloes dominated galaxy formation at this early epoch.

In summary, the velocity effect makes it significantly easier to detect the 21-cm power spectrum during the epoch of the first heating of the IGM. It also creates a clear signature by flattening the power spectrum and increasing the prominence of the BAOs (which are more strongly imprinted in the velocity than in the density fluctuations). While Figure 10 considers a single redshift, similar observations over the full $\Delta z \sim 6$ redshift range of significant heating fluctuations could actually detect the slow advance of the LW feedback process, 


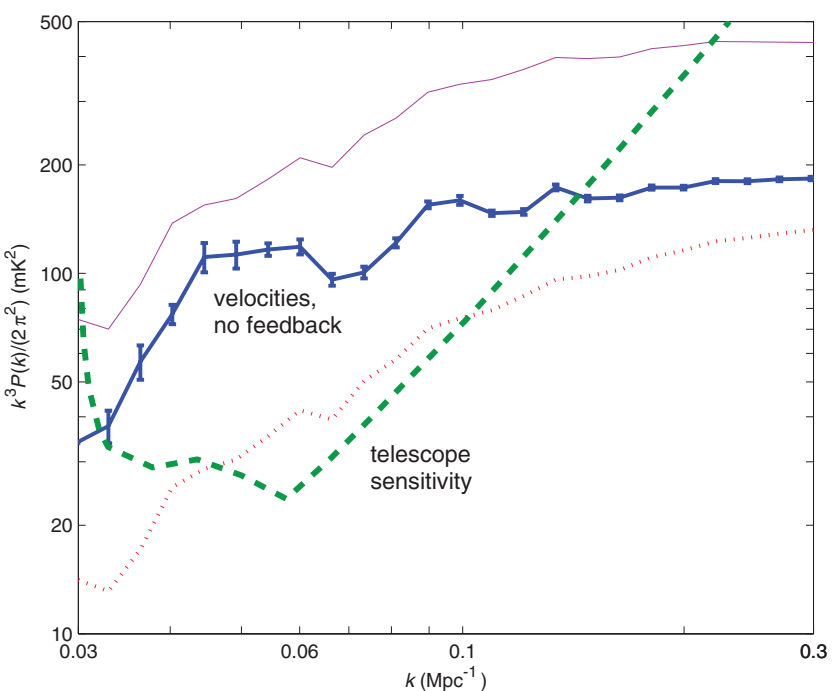

Figure 10. Signature of the relative velocity in the $21-\mathrm{cm}$ power spectrum, at the peak of the X-ray heating transition at $z=20$. We consider the prediction including the relative velocity effect (blue solid curve) or with the effect of densities only (red dotted curve), both for the case of a late LW transition for which the LW feedback is still negligible at $z=20$. These predictions are compared to the projected $1 \sigma$ telescope sensitivity (green dashed curve) based on 1000-h observations with an instrument such as the Murchison Wide-field Array or the Low Frequency Array but designed to operate at $50-100 \mathrm{MHz}$ (McQuinn et al. 2006), where we include an estimated degradation factor due to foreground removal (Liu 2012); this sensitivity is defined as the signal that would yield a measurement with a signal-to-noise ratio of unity in each $k$ bin of size $\Delta k=0.5 k$ averaged over an 8-MHz frequency band. Future experiments such as the Square Kilometre Array should reach a better sensitivity by more than an order of magnitude (McQuinn et al. 2006). To allow for the possibility of feedback, we also show the prediction for the opposite limit of maximum feedback, i.e., an early LW transition that has already saturated (purple solid curve). In this plot, we have fixed the heating transition at $z=20$ for easy comparison among the various cases. From Visbal et al. (2012).

during which the power spectrum is predicted to continuously change shape, gradually steepening as the BAO signature weakens towards low redshift.

\section{OPEN QUESTIONS}

As summarised above, the recently noticed velocity streaming effect causes a special form of luminosity bias at high redshift. Unlike the standard galaxy bias relative to the underlying density field, early galaxies were subject to a bias that depends on the velocity field. Specifically, the velocity difference between the baryons and dark matter affected the smallest star-forming haloes in which the gas cooled via molecular hydrogen cooling. This bias of luminosity relative to velocity results in large fluctuations in the abundance of the first stars. Since the velocity varies on larger scales than the density field, and larger scales are easier to observe (since lower resolution suffices to detect the corresponding $21-\mathrm{cm}$ fluctuations), the velocity effect immensely increases the feasibility for upcoming 21-cm observations to detect stars from higher redshifts $(\sim 15-25)$ than previously expected. More- over, such a detection would be both easier to recognise and more exciting than previously thought.

These developments should make the whole prereionisation epoch far more attractive and interesting to the whole community of theorists, numerical simulators, and observers. In fact, this era is rich with little-explored astrophysics. As the first stars formed, their radiation (plus emission from stellar remnants) produced feedback that radically affected not only the IGM but also the character of newly forming stars. As shown above, the velocity effect substantially modifies the distribution of the first stellar generations, and in fact the star formation history is changed further since radiative feedback (which affects all stars beyond the very first ones) itself depends on this distribution.

In the previous section, we have mentioned only some first explorations of the radiative feedback during this early era. However, in order to more reliably guide observers to the expected $21-\mathrm{cm}$ power spectrum from this era, the radiative feedback should be explored in much greater detail. For example, the 21-cm fluctuations generated during the heating transition, studied by Pritchard \& Furlanetto (2007) without the streaming velocity and by Visbal et al. (2012) with the velocity included, should be studied together with inhomogeneous LW feedback. Also, the 21-cm fluctuations during Ly $\alpha$ coupling originally studied by Barkana \& Loeb (2005b) should be studied in the presence of the streaming velocity and the LW feedback. A full study of these potentially observable signals will also require a new generation of numerical simulations that will explore the small-scale interaction between relative velocity and star formation in the presence of various feedbacks such as heating and LW feedback.

At the same time, significant uncertainty is likely to always remain in any prediction that involves complex astrophysics such as star formation and feedback. Thus, only observations can ultimately confirm or exclude the promising theoretical predictions shown in the previous section. We therefore expect increased observational efforts focused on this early epoch. Such observations would push well past the current frontier of cosmic reionisation $(z \sim 10, t \sim 480 \mathrm{Myr})$ for galaxy searches and $21-\mathrm{cm}$ arrays, and thus would represent a new frontier for extragalactic astronomy.

\section{ACKNOWLEDGEMENTS}

I thank my collaborators on the reviewed work, especially Anastasia Fialkov, Eli Visbal, Dmitriy Tseliakhovich, Chris Hirata, Avi Loeb, and Smadar Naoz. This work was supported by Israel Science Foundation grant 823/09.

\section{REFERENCES}

Abel, T., Bryan, G. L., \& Norman, M. L. 2002, Sci, 295, 93

Ade, P. A. R., et al. (Planck Collaboration) 2013, A\&A, in press (arXiv:1303.5076)

Aihara, H., et al. 2011, ApJS, 193, 29 (erratum: ApJS, 195, 26) 
Ali, S. S., Bharadwaj, S., \& Pandey, B. 2005, MNRAS, 363, 251

Bardeen, J. M., Bond, J. R., Kaiser, N., \& Szalay, A. S. 1986, ApJ, 304, 15

Barkana, R. 2006a, MNRAS, 372, 259

Barkana, R. 2006b, Sci, 313, 931

Barkana, R. 2009, MNRAS, 397, 1454

Barkana, R., Haiman, Z., \& Ostriker, J. P. 2001, ApJ, 558, 482

Barkana, R., \& Loeb, A. 2004, ApJ, 609, 474

Barkana, R., \& Loeb, A. 2005a, ApJL, 624, 65

Barkana, R., \& Loeb, A. 2005b, ApJ, 626, 1

Bharadwaj, S., \& Pandey, S. K. 2005, MNRAS, 358, 968

Bond, J. R., Cole, S., Efstathiou, G., \& Kaiser, N. 1991, ApJ, 379, 440

Bond, J. R., Kofman, L., \& Pogosyan, D. 1996, Natur, 380, 603

Bowman, J. D., Morales, M. F., \& Hewitt, J. N. 2007, ApJ, 661, 1

Bowman, J. D., Morales, M. F., \& Hewitt, J. N. 2009, ApJ, 695, 183

Bowman, J. D., \& Rogers, A. E. E. 2010, Natur, 468, 796

Bromm, V., Coppie, P. S., \& Larson, R. B. 1999, ApJ, 527, L5

Chen, X., \& Miralda-Escudé, J. 2004, ApJ, 602, 1

Chuzhoy, L., Alvarez, M. A., \& Shapiro, P. R. 2006, ApJ, 648, L1

Ciardi, B., Ferrara, A., \& White, S. D. M. 2003, MNRAS, 344, L7

Cole, S., \& Kaiser, N. 1989, MNRAS, 237, 1127

Colless, M., et al. 2001, MNRAS, 328, 1039

Curtis-Lake, E., et al. 2013, MNRAS, 429, 302

Dalal, N., Pen, U.-L., \& Seljak, U. 2010, JCAP, 11, 7

Fialkov, A., Barkana, R., Tseliakhovich, D., \& Hirata, C. 2012, MNRAS, 424, 1335

Field, G. B. 1958, Proc. IRE, 46, 240

Furlanetto, S. R., Oh, S. P., \& Briggs, F. H. 2006, PhR, 433, 181

Furlanetto, S. R., Zaldarriaga, M., \& Hernquist, L. 2004a, ApJ, 613, 1

Furlanetto, S. R., Zaldarriaga, M., \& Hernquist, L. 2004b, ApJ, 613, 16

Gardner, J. P., et al. 2006, SSRv, 123, 485

Greif, T., White, S., Klessen, R., \& Springel, V. 2011, ApJ, 736, 147

Gunn, J. E., \& Peterson, B. A. 1965, ApJ, 142, 1633

Haiman, Z., Rees, M. J., \& Loeb, A. 1997, ApJ, 476, 458 (erratum: ApJ, 484, 985)

Harker, G. 2010, MNRAS, 405, 2492

Hinshaw, G., et al. 2012, ApJS, in press (arXiv:1212.5226)

Hirata, C. M. 2006, MNRAS, 367, 259

Hogan, C. J., \& Rees, M. J. 1979, MNRAS, 188, 791

Ichikawa, K., Barkana, R., Iliev, I. T., Mellema, G., \& Shapiro, P. R. 2010, MNRAS, 406, 2521
Kaiser, N. 1984, ApJ, 284, L9

Liu, A., \& Tegmark, M. 2012, MNRAS, 419, 3491

Loeb, A., \& Zaldarriaga, M. 2004, PRL, 92, 211301

Madau, P., Meiksin, A., \& Rees, M. J. 1997, ApJ, 475, 429

Maio, U., Koopmans, L. V. E., \& Ciardi, B. 2011, MNRAS, 412, L40

McQuinn, M., \& O'Leary, R. M. 2012, ApJ, 760, 3

McQuinn, M., Zahn, O., Zaldarriaga, M., Hernquist, L., \& Furlanetto, S. R. 2006, ApJ, 653, 815

Mellema, G., Iliev, I. T., Pen, U.-L., \& Shapiro, P. R. 2006, MNRAS, 372, 679

Mesinger, A., Furlanetto, S., \& Cen, R. 2011, MNRAS, 411, 955

Mo, H. J., \& White, S. D. M. 1996, MNRAS, 282, 347

Morandi, A., \& Barkana, R. 2012, MNRAS, 424, 2551

Naoz, S., \& Barkana, R. 2008, MNRAS, 385, 63

Naoz, S., Noter, S., \& Barkana, R. 2006, MNRAS, 373, L98

Naoz, S., Yoshida, N., \& Gnedin, N. Y. 2012, ApJ, 747, 128

Naoz, S., Yoshida, N., \& Gnedin, N. Y. 2013, ApJ, 763, 27

Nusser, A. 2005, MNRAS, 364, 743

O'Leary, R. M., \& McQuinn, M. 2012, ApJ, 760, 4

Ono, Y., Shimasaku, K., Dunlop, J., Farrah, D., McLure, R., \& Okamura, S. 2010, ApJ, 724, 1524

Ono, Y., et al. 2012, ApJ, 744, 83

Paciga, G., et al. 2011, MNRAS 413, 1174

Pan, T., \& Barkana, R. 2012, arXiv:1209.5751

Parsons, A. R., et al. 2010, AJ, 139, 1468

Press, W. H., \& Schechter, P. 1974, ApJ, 187, 425

Pritchard, J. R., \& Furlanetto, S. R. 2006, MNRAS, 367, 1057

Pritchard, J. R., \& Furlanetto, S. R. 2007, MNRAS, 376, 1680

Pritchard, J. R., \& Loeb, A. 2010, PRD, 82, 023006

Robertson, B. E., et al. 2013, ApJ, 768, 71

Santos, M. G., Amblard, A., Pritchard, J., Trac, H., Cen, R., \& Cooray, A. 2008, ApJ, 689, 1

Schaerer, D., \& de Barros, S. 2010, A\&A, 515, A73

Scott, D., \& Rees, M. J. 1990, MNRAS, 247, 510

Semelin, B., Combes, F., \& Baek, S. 2007, A\&A, 474, 365

Springel, V., Frenk, C. S., \& White, S. D. M. 2006, Natur, 440, 1137

Stacy, A., Bromm, V., \& Loeb, A. 2011, MNRAS, 413, 543

Tseliakhovich, D., Barkana, R., \& Hirata, C. 2011, MNRAS, 418, 906

Tseliakhovich, D., \& Hirata, C. M. 2010, PRD, 82, 083520

Visbal, E., Barkana, R., Fialkov, A., Tseliakhovich, D., \& Hirata , C. M. 2012, Natur, 487, 70

Wouthuysen, S. A. 1952, AJ, 57, 31

Zahn, O., Lidz, A., McQuinn, M., Dutta, S., Hernquist, L., Zaldarriaga, M., \& Furlanetto, S. R. 2007, ApJ, 654, 12 